\title{
Hacia una epistemología de la emergencia tecnológica en la educación
}

Flor de Liz Pérez Morales*, Rosaura Castillo Guzmán**, Angélica María Fabila Echauri***

DOI: https://doi.org/:10.33571/revistaluciernaga.v12n24a2

\section{Resumen}

Se discute la llamada <<emergencia tecnológica〉>, utilizando como método el debate epistemológico de la perspectiva de lo emergente para comprender los escenarios tecnológicos. Esta forma de concebirla pone en el centro del debate la relación propia entre la tecnología y la educación, así como la noción de emergencia, lo que permite entender la correlación de fuerzas que produce un acontecimiento social, disruptivo que dibuja una crisis y que denominamos emergencia social.

La emergencia es un concepto que explora alternativas a las nuevas condiciones de la realidad social; de manera natural no adivina los derroteros de la sociedad, aunque busque anticipar los riegos, planteando las vulnerabilidades. El rumbo inmediato se orienta a crear una cultura para atender la emergencia; pero también está develando el proceder humano prioritariamente en la instancia de la ética, un rumbo que está poniendo en el debate los mecanismos formativos de la educación y la tecnología sobre razones más profundas como la sobrevivencia.

Palabras claves: Emergencia tecnológica; educación; epistemología.

Recibido. Junio 21, 2020

Aceptado. Julio 28, 2020

* Profesora-Investigadora de la DAEA- UJAT. Doctora en Estudios Transdisciplinarios en Cultura y Comunicación (ICONOS), Maestra en Docencia (UJAT), Licenciada en Ciencias de la Comunicación (UAM-X), Especialidad en Administración Pública. Candidata a SIN. Orcid: https://orcid.org/0000-0001-6478-3727; e-mail: fplizliz@gmail.com _

** Profesora - investigadora de la Universidad Juárez Autónoma de Tabasco, en la División Académica de Educación y Artes. Estudios Realizados: Licenciada en Ciencias de la Educación, especialidad en docencia y la Maestría en Docencia por la Universidad Juárez Autónoma de Tabasco, Doctorado en Educación por CESU-OLMECA. Orcid: https://orcid.org/00000003-0441-5484 ; e-mail: rossycastillo 65@hotmail.com

*** Profesora - investigadora de la Universidad Juárez Autónoma de Tabasco, en la División Académica de Educación y Artes. Licenciada en Comunicación por la Universidad Autónoma Metropolitana-Xochimilco. Maestra en Tecnología Educativa por el Centro de Excelencia de la Universidad Autónoma de Tamaulipas. Doctora en Comunicación Educativa, por la Universidad de Baja California. Orcid: https://orcid.org/0000-0001-5667-3712 ; e-mail: angelifa@hotmail.com 


\title{
Towards an epistemology of technological emergence in education
}

Flor de Liz Pérez Morales*, Rosaura Castillo Guzmán**, Angélica María Fabila Echauri***

DOI: https://doi.org/:10.33571/revistaluciernaga.v12n24a2

\begin{abstract}
This reflection discusses the so-called <<technological emergence $>>$, by using the epistemological debate of the emergent perspective as a method to understand technological scenarios. This way of conceiving it is focused on the relationship between technology and education, as well as on the notion of emergence, which allows us to understand the correlation of forces that produces a social, disruptive event that draws a crisis called social emergence.
\end{abstract}

Emergency is a concept that explores alternatives to the new conditions of social reality; it does not naturally predict the course of society, although it seeks to anticipate the risks, raising vulnerabilities. The immediate course is oriented to create a culture to face the emergency; but it is also unveiling the human proceeding primarily in the instance of ethics, a course that is setting in the debate the formative mechanisms of education and technology on deeper reasons such as survival.

Keywords: Technological emergence; education; epistemology.

Received. June 21, $2020 \quad$ Accepted. July 28, 2020

* Professor-Researcher at DAEA- UJAT. PhD in Transdisciplinary Studies in Culture and Communication (ICONOS), Master in Teaching (UJAT), Bachelor in Communication Sciences (UAM-X), Specialty in Public Administration. Candidate for SIN. Orcid: https://orcid.org/0000-0001-6478-3727 ; e-mail: fplizliz@gmail.com

** Professor - researcher at the Universidad Juárez Autónoma de Tabasco, in the Academic Division of Education and Arts. Education: Bachelor's Degree in Education Sciences, specializing in teaching and Master's Degree in Teaching from the Universidad Juárez Autónoma de Tabasco, Doctorate in Education from CESU-OLMECA. Orcid: https://orcid.org/0000-00030441-5484 e-mail: rossycastillo_65@hotmail.com

*** Professor - researcher at Universidad Juárez Autónoma de Tabasco, in the Academic Division of Education and Arts. B.A. in Communication from the Universidad Autónoma Metropolitana-Xochimilco. Master in Educational Technology from the Center of Excellence of the Autonomous University of Tamaulipas. PhD in Educational Communication from Universidad de Baja California. Orcid: https://orcid.org/0000-0001-5667-3712 ; e-mail: angelifa@hotmail.com

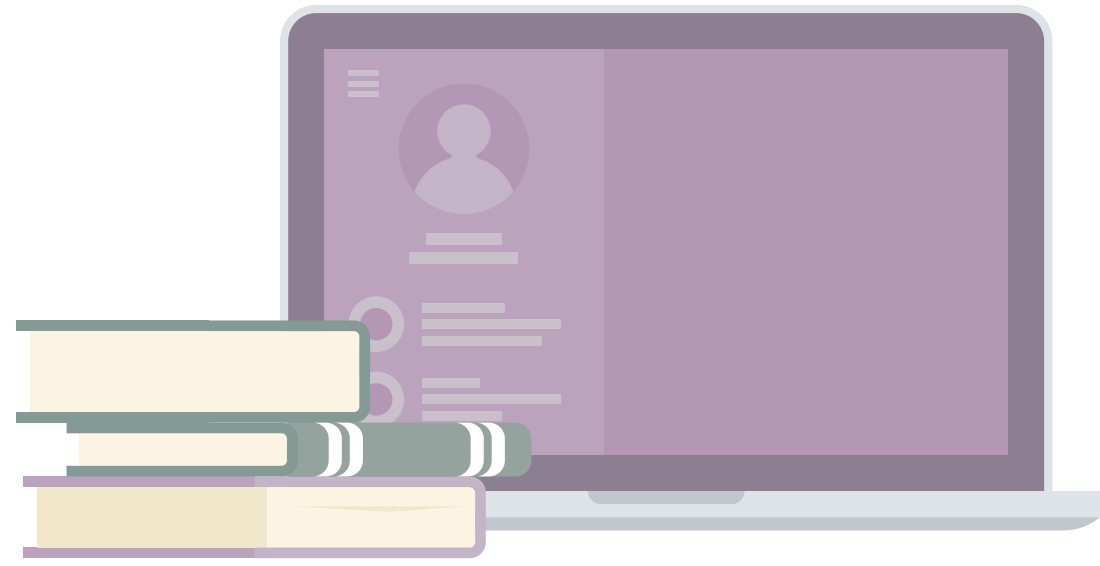




\section{Rumo a uma epistemologia de emergência tecnológica na educação}

Flor de Liz Pérez Morales*, Rosaura Castillo Guzmán**, Angélica María Fabila Echauri***

DOI: https://doi.org/:10.33571/revistaluciernaga.v12n24a2

\section{Resumo}

Esta reflexão discute a chamada <<emergência tecnológica>>, colocando como método o debate epistemológico da perspectiva do emergente para compreender os cenários tecnológicos. Esta forma de conceber coloca no centro do debate a relação entre tecnologia e educação, bem como a noção de emergência, o que permite compreender a correlação de forças que produz um acontecimento social, perturbador, que desenha uma crise e que chamamos de emergência social.

A emergência é um conceito que explora alternativas às novas condições da realidade social; de maneira natural não prediz os caminhos da sociedade, embora procure antecipar os riscos, colocando as vulnerabilidades. O curso imediato está orientado para criar uma cultura para atender à emergência; mas também está a revelar o procedimento humano principalmente na instância da ética, um curso que está a colocar no debate os mecanismos formativos da educação e da tecnologia sobre razões mais profundas como a sobrevivência

Palavras chave: Emergência tecnológica; educação; epistemologia.

Recebido. Junho 21, 2020

Aceitado. Julho 28, 2020

* Professor-Pesquisador no DAEA- UJAT. Doutoramento em Estudos Transdisciplinares em Cultura e Comunicação (ICONOS), Mestrado em Ensino (UJAT), Bacharelato em Ciências da Comunicação (UAM-X), Especialidade em Administração Pública. Candidato ao SIN. Orcid: https://orcid.org/0000-0001-6478-3727 ; e-mail: fplizliz@gmail.com

** Professor - investigador na Universidade Juárez Autónoma de Tabasco, na Divisão Académica de Educação e Artes. Estudos: Licenciatura em Ciências da Educação, especialização em ensino e Mestrado em Ensino da Universidade Juárez Autónoma de Tabasco, Doutoramento em Educação pelo CESU-OLMECA. Orcid: https://orcid.org/0000-0003-0441-5484; e-mail: rossycastillo_65@hotmail.com

*** Professor - investigador na Universidade Juárez Autónoma de Tabasco, na Divisão Académica de Educação e Artes. Licenciado em Comunicação pela Universidad Autónoma Metropolitana. Orcid: https://orcid.org/0000-0001-5667-3712; e-mail: angelifa@hotmail.com

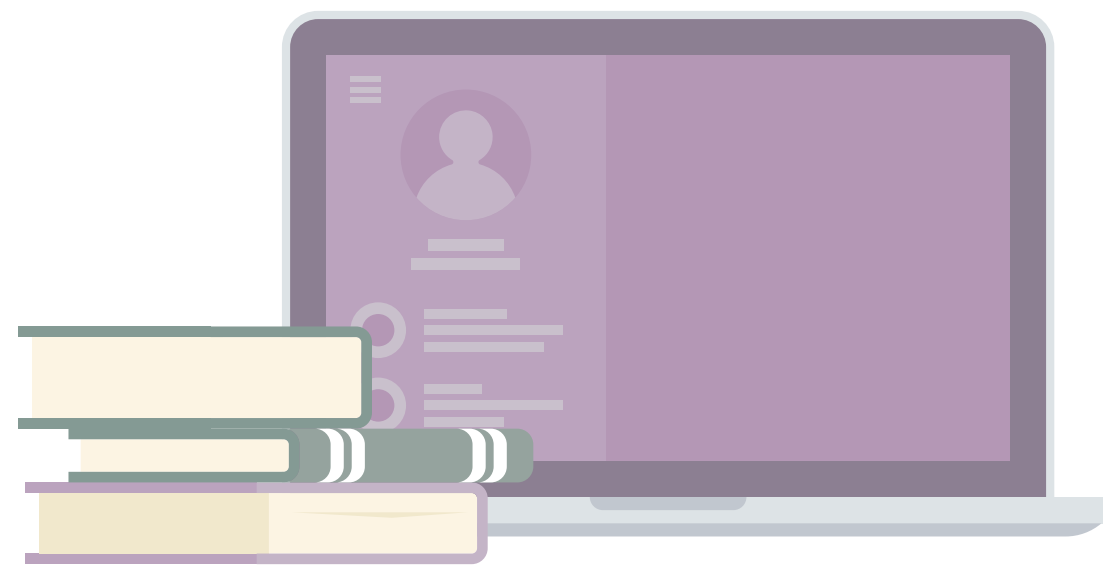




\section{Introducción}

Cuando el escocés Robert Louis Stevenson escribió su obra El extraño caso del doctor Jekyll y mister Hyde (1886), nunca se imaginó que con ella estaba también describiendo una alegoría del futuro; el extraño caso de un mismo cuerpo con dos perfiles; una metáfora muy recurrente de las polaridades o paradojas que ahora mismo se tienen con el recurso tecnológico en la educación; una mirada dual que se acomoda y reacomoda; a veces enseñando su mejor cara, o por el contrario mostrando las deformaciones que se visibilizan en los cambios del mundo contemporáneo; un universo poblado de circunstancias adversas que inquiere de mejores respuestas. Tras ello, lo que se aguarda de este rostro dual del conocimiento sobre tecnología-educación es la promesa hecha desde la modernidad de una humanidad con bienestar social; el mundo <<feliz>> de muchos autores.
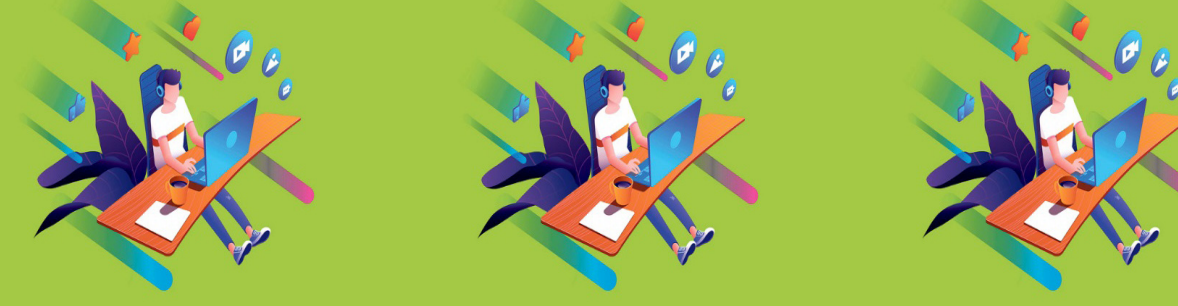

El mundo contemporáneo, en su inestabilidad, transformaciones, vulnerabilidad y acelerado modo de vida, se sitúa en condiciones que apuntan hacia el deterioro de nuestra vida en sus entornos vitales; campos de emergencias [1], que tienen la particularidad de ser diversos y múltiples al mismo tiempo. La orientación de esfuerzos para subsanar los campos de tensión que se dirimen en ciudades y localidades se ha encaminado a solventar o minimizar los riesgos, paliativos que no definen profesiones, pero que sin duda demandan y multiplican los esfuerzos de muchas áreas del conocimiento.

Es un hecho que las transformaciones en el mundo guardan una íntima relación entre el pensamiento humano y las condiciones de vida en las que se insertan. "Los objetos, los instrumentos y los artefactos no son algo externo a nosotros; forman parte de nosotros, y construimos nuestra vida, nuestro conocimiento y nuestra identidad a partir de las relaciones que establecemos con ellos" (Gros, 2008: 10). Lo que ahora mismo acontece con las mudanzas de la humanidad es inherente a los procesos tecnológicos que dinamizan su vida social, explicaciones que se colocan como rasgo del progreso. Daniel Jacques dice que la revolución técnica que se vive arrastra a las otras (revoluciones) que le precedieron, porque integra en sí misma las tareas que antes se les encomendaban a otros sectores (2003).

Las rutas tomadas son alicientes que mitigan muy poco los marcos críticos que se viven. Sobre estos quiebres sociales, y por ende de resistencia de las comunidades afectadas, dos ámbitos fundamentales han sido llamados para la atención de estas transformaciones mundiales y locales; sin menoscabo de otros, el ánimo esperanzador ha recaído sobre la educación en su vínculo o relación con la tecnología. 
El carácter que presenta el mundo de hoy no puede ser evadido ni por la educación ni por la tecnología; por el contrario, la incidencia de ambas en los tiempos actuales puede alcanzar razones válidas para colocarlas como trabes que soporten lo caótico de las experiencias vividas por los pueblos o ciudades.

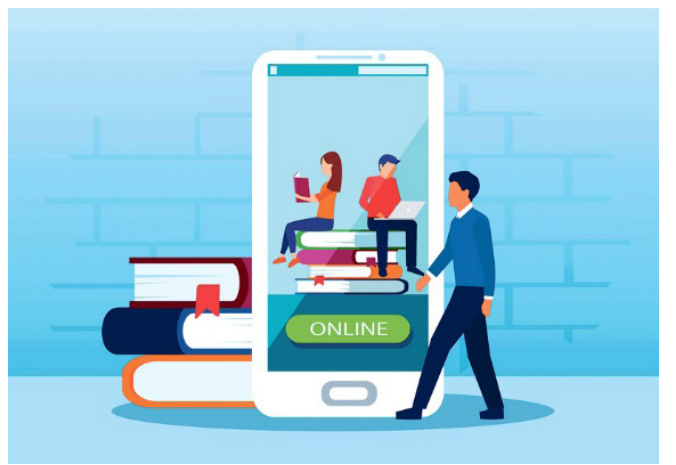

La tecnología se constituye entonces como un elemento característico de nuestro tiempo; reflexionarla en el marco de su incidencia en el terreno educativo presume al menos dos formas de entenderla: las más recurrente de ellas es caracterizada por el reclamo de su incorporación, llamémosle su emergencia, en los procesos educativos a fin de hacerlos más eficientes como consecuencia de sus bondades.

En paralelo, la segunda perspectiva, menos socorrida, es la mirada de una tecnología que se constituye, más que en un medio, en un escenario emblemático de nuestras sociedades, donde la educación es convocada para intervenir y atender las emergencias que este escenario funda en los procesos formativos y en múltiples actividades humanas.

La paradoja se asienta entonces en la vertiente de que la tecnología debe proporcionar alicientes a la educación, o la otra vertiente, donde la educación debe ser un aliciente para la crisis que provoca el escenario tecnológico. En la primera arista, Baudrillard menciona que la tecnología es vista en su forma heroica. Por el contrario, los excesos de confianza en ella, y su ilusión o seducción, aduce a la segunda forma, a la que denomina irónica (2010: 62). Sin embargo, Baudrillard aclara que, en su forma irónica, "la ilusión no es un error o una decepción, sino un juego, un gran juego cuyas reglas desconocemos y las cuales quizá, nunca conozcamos" (2010: 65). Ambas rutas se tornan complejas, pues el valor ético y responsable de las dos entra en conflicto en el terreno de la emergencia. El incuestionable punto crítico que deviene en el juego de las responsabilidades es justamente lo que genera el objetivo inicial de esta preocupación.

La incidencia que proyectan la tecnología y la educación está demarcando una ruta que coloca el debate de la emergencia en los ojos de una discusión elemental entre ellas. Los análisis brindan algunas claves de comprensión, pero no asuntan en las salidas adecuadas para los estudios de los escenarios emergentes. No se ha podido evitar que el mundo siga encaminándose hacia su desbocamiento como bien menciona Anthony Giddens (2007).

Ante este mundo complejo-emergente se detecta entonces la necesidad de contextualizar estos nuevos escenarios. Realizamos un diagnostico con los datos obtenidos de foros con estudiantes, la revisión de políticas públicas y los planteamientos descritos en los planes de 
estudio de la formación en comunicación de la Universidad Juárez Autónoma de TabascoUAJT- México.

La investigación comenzó desde el año 2007 y tuvo los primeros acercamientos al plan de estudio de la licenciatura en Comunicación de 2010 con la integración del campo disciplinar de Comunicación Emergente (UJAT, 2010). En el más reciente plan de estudios de este pregrado (UJAT, 2018) se opera la línea curricular Comunicación Emergente, con avances teóricos y metodológicos que se conciben con asignaturas y proyectos de investigación en esta línea para incidir en el curriculum desde la práctica educativa.

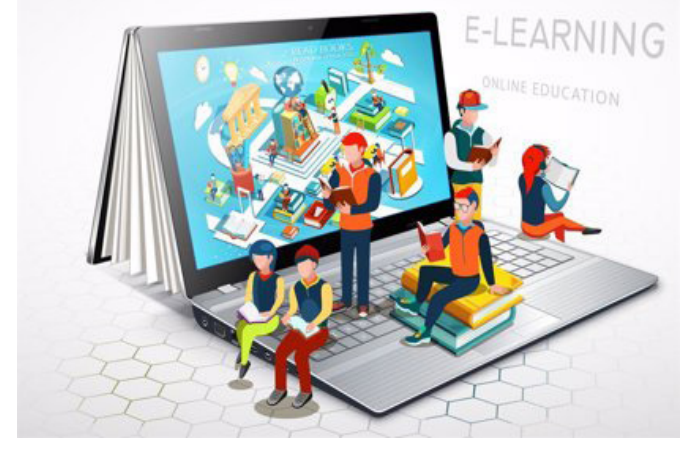

\section{Desarrollo}

\subsection{De la emergencia educativa a la tecnología}

Solícitas de mejores aprendizajes para la formación, las instituciones educativas se han embarcado en el tren de la heroicidad tecnológica. En esta perspectiva la tecnología es un medio que debe favorecer el aprendizaje de conocimientos significativos para los estudiantes. El recurso tecnológico es un medio para llegar a ellos; sin embargo, la promesa no siempre se entiende de esa manera. El aprendizaje se ha encargado a las potencialidades absolutas del recurso técnico; la escuela ha asumido esto como una necesidad donde no se puede estar alejado de ella. El problema se entona desde que se entiende el uso vital de la tecnología como "una moda", y no como una mejor forma de acercarse al conocimiento, y por ende a los aprendizajes.

A menudo, los educadores parecen conformarse con la reivindicación del acceso de los estudiantes a las tecnologías. Pero no se trata sólo de acceder a ellas y obtener información, sino de verificar, construir, validar, crear conocimientos e interaccionar con los otros. La vida se transforma a partir del uso de los medios; no podemos ser sólo consumidores, sino que debemos tratar de mejor nuestras habilidades y competencias (Gros, 2008: 10)

El mundo actual exige otras habilidades y capacidades educativas de los sujetos; uno donde las formaciones estén a la par de sus circunstancias. Desde aquí, las necesidades urgentes de la educación son las que reclaman a la tecnología las potencialidades de su uso. "La tecnología se convierte en una herramienta cognitiva que forma parte del propio proceso de aprendizaje" (Gros, 2008: 11). 
Este reclamo debe auspiciar procesos claros de la tecnología como un medio que auxilie en los aprendizajes, tales como: metodologías y métodos que favorezcan la construcción del conocimiento, y herramientas virtuales que detonen apuestas y respuestas adecuadas a los problemas educativos. Es esa formación para el mundo que centra su especificidad en una urgente transformación de sus procesos educativos, y donde la tecnología tiene cabida como un recurso educativo para adiestrar al sujeto sobre mejores formas aprendizajes.

En esencia, desde aquí se trata de entender que la educación, como un proceso dinámico cuya naturaleza cotidiana conlleva innovaciones constantes, convoca a la tecnología como un apoyo ineludible que puede ayudar a procesar otras habilidades y capacidades en los sujetos de aprendizaje. El objeto tecnológico es imaginado por la escuela para solventar una necesidad pedagógicamente requerida.

Aquí la tecnología sólo es vista como mero recurso técnico que "higiénicamente" permite el adiestramiento técnico de un sujeto que puede llevarla a su aprovechamiento funcional y social. En este ángulo la tecnología no parece causar ningún daño, por el contrario, su abordaje, como menciona Baudrillard, se carga de heroicidad cuyas bondades producirán el mejor beneficio educativo.

Muchos son los compromisos que las escuelas (públicas y privadas) asumen respecto de lo tecnológico; las promesas se venden como innovaciones educativas que brindaran la mejor formación de los estudiantes. Pocos se preguntan si tales ofrecimientos son reales y si estas estrategias serán un verdadero soporte de conocimientos para los alumnos. El ofrecimiento de una tableta o el acceso a internet, no ha significado una garantía, un aprendizaje adecuado para las instituciones y por consecuencia para la sociedad.

En el caso de México, por ejemplo, el acceso tecnológico para los planteles educativos se ha dificultado de muchas formas; desde la infraestructura, los modelos y programas educativos, hasta la administración y docencia educativa. Se suma la pobreza y marginalidad de muchas escuelas, pero también las capacidades y habilidades de los actores que se resisten a reconocer la necesidad de adiestrar pedagógicamente a los educandos para sobrevivir en el universo tecnológico. La gestión y la falta de transparencia en los procesos académicos han llevado a la creación de espejismos educativos, donde la tecnología parecería resolver todos los problemas de fondo, desde lo formativo, hasta la incidencia tecnológica en la sociedad. El asunto es que la educación apremia una emergencia, que no sólo la pone en el debate de la brecha tecnológica, sino de la engañosa mirada de la tecnología en las didácticas institucionales.

Justo aquí se coloca una de las interrogantes y preocupaciones más agudas para la educación; un espectro que se torna complejo y su tarea complicada, lo que sin lugar a dudas implicaría la recomposición de la educación en todas sus dimensiones (institución escolar, modelos educativos, currículos, practica tecno-educativa, institución familiar, gobiernos, políticas públicas, etc.) reflexión que, aunque no corresponde a este análisis, tampoco significa su deslinde. Lo cierto es que la misma ruta está demarcando bemoles que se convierten en problemas, que abren muchas interrogantes aun cuando se entiende la importancia que las tecnológicas cobran en la educación. 
El problema se cierne cuando sólo se mira a la tecnología <<ingenuamente>>, sin posibilidad de cruzar los límites de su propia intención ética-humana; cuando se deja de entender como portadora también de una intención simbólica, que carga un "saber arqueológico", cuyo devenir histórico también llega al sujeto (Lizarazo, 2007).

Su dominio en el marco subjetivo e intersubjetivo de los sujetos de aprendizaje se establece como referencia social. Uno donde la tecnología se yergue con un reinado sobre el que se formulan y se concentran todas las visiones del mundo. Las pedagogías, a través de los currículos escolares se trazan sobre esa ruta. "Se trata de una pedagogía porque su oriente es propiciar la comprensión, y dicha comprensión es el aprendizaje de sentido. (Lizarazo, 2007: 18). Refiere que no sólo se trata del aprendizaje epistémico, sino también en lo ético, social y humano. Sin la alerta que esto produce, se entra al campo minado de la emergencia tecnológica, donde las tecnologías dejan de tener sus rasgos bondadosos y puede mostrar el otro rostro: el de la crisis.

\subsection{De la emergencia tecnológica a la educación}

No se puede negar la incidencia de la tecnología en la historia y el presente del mundo. El siglo XXI trajo consigo el recurso tecnológico más icónico de la vida actual como lo es el internet, símbolo de una mudanza paulatina del territorio y la habitabilidad de los sujetos. El ciberespacio procreó redes y multitudes conectadas que, si bien visibilizan y acercan a otras fuentes de conocimiento, también es cierto que <<purgan>> con las inconsistencias sociales de nuestro tiempo. El mundo conformado en las redes sociales se hizo comunal, performativo, discontinuo e intenso.

Producto de la incursión tecnológica sin límites nos hemos visto inmersos en un territorio desconocido, sin estar preparados para él. El cibermundo arribó a todos los ámbitos sociales y creó la otra <<ciberhumanidad>> como una prolongación desconocida de la vida humana; las herramientas y las máquinas se implantaron, y con ellas se suple cotidianamente, con tal intensidad y celeridad, lo humano. Se despliega un movimiento sin cortapisas que difícilmente se sabe por dónde camina. Atraviesa todos los linderos, brama su propia potencia como hallazgos de todo progreso social. Las metáforas que anunciaban una técnica que nombró a la ciencia como su protectora, estimuló el universo de lo fatuo, lo que ha dejado al descubierto que: frente al poder tecnológico, lo humano también se hace vulnerable, falible e incierto.

El mundo apocalíptico destruido por la tecnología y dibujado por muchos autores en la literatura, el cine y las artes en general, procrea las alertas educativas. Los cangrejos caminan sobre las islas (1958), de Anatoli Dneprov o Blade Runner (1992) de Ridley Scott, colocan a la tecnología sobre esa sospecha. Jean Baudrillard, da cuenta de ello:

La humanidad no discrimina; de buen agrado se convierte en su propio conejillo de indias bajo los mismos términos que el resto del mundo animado e inanimado. La humanidad no juega alegremente su propio futuro como especie, de la misma forma que se juega el futuro de todas las demás criaturas. En su 
búsqueda ciega para alcanzar un mayor conocimiento, la humanidad programa su propia destrucción con la misma ferocidad casual con la que se aplica en la destrucción de todo lo demás. La humanidad se sacrifica como especie a un destino experimental desconocido: en cualquier caso, para otras especies, que nunca han conocido ningún destino salvo el natural (2010: 25).

El peregrinaje de lo tecnológico siembra muchas inquietudes en todos los ámbitos; la fe ciega en la tecnología apunta a su propia desconfianza y avizora sobre sus riesgos; para su ayuda la mirada se fija en las posibilidades de la educación, coloca su emergencia en su condición ética, auxilio de los devenires anunciados. La herramienta tecnológica trasciende a un disruptivo escenario crítico, donde la educación es invitada a socorrerle. Es desde ahí donde la educación es convocada para intervenir en el tejido de la crisis de la tecnología que deviene en una complejidad y por tanto acuña sus propios cánones. Esta dualidad procrea un conflicto social que detona en una emergencia, donde los imperativos de la tecnología rebasan muchas fronteras sociales.

La lógica de lo tecnológico y las mutaciones de la vida social incitan, desde el conocimiento, múltiples posibilidades de comprensión. Por ejemplo, en el territorio de lo virtual, dice Baudrillard, se introduce lo transhistórico o lo transpolítico; es decir, la esfera donde el tiempo real tiene otros significados:

...precisamente, porque se producen y se emiten <<en tiempo real >>, donde no tienen significado, porque tienen todos los significados posibles. Por consiguiente, tenemos que comprenderlos no políticamente, sino transpolíticamente; es decir, en el punto donde se pierden en el vacío de información. La esfera de información es un espacio donde los acontecimientos, desprovistos de su significado, reciben una gravedad artificial, donde, después de haber sido congelados política e históricamente, se vuelven a organizar transpolíticamente, en tiempo real, es decir, perfectamente virtual. Podríamos hablar de la misma forma de la esfera transeconómica (2010: 61).

Baudrillard explica que un acontecimiento tecnológico ya no tiene significado por sí mismo, el acontecimiento producido por la información ya no tiene significado histórico por sí mismo. El mismo autor señala que la tecnología ha roto con muchos planteamientos claros y con muchos esquemas que han ido más allá de sus límites, se han movido más allá de las leyes de la física y de la metafísica (2010).

Lo que se deja en claro entonces no es la perspectiva de la relación tecnología-educación, sino la condición de emergencia que vive nuestro mundo. Una alerta que, como en muchos otros espacios sociales, comienza a verter sus estragos. El problema que también se desnuda es que aun cuando se localiza un objeto de estudio emergente, su abordaje demanda diversas áreas del conocimiento.

El mundo tecnológico hace emerger otros conflictos que modifican los conceptos, los mapas y las rutas del conocimiento. Las sociedades transitaron a la <<comunalidad $>>$; espacios 
donde los signos sociales alegan los síntomas de una sociedad en crisis. Ahí se hacen las nuevas guerras con las protestas sociales, la volatilización de los mercados, la cristalización de la política, la <<viralización>> del acontecimiento, pero también los deseos y utopías mundiales. Desde este territorio se alude a una condición propia de la emergencia que se vuelca en una perspectiva de estudio que, sin dudarlo, trae consigo la complejidad misma del término.

Se abren aquí dos interrogantes que debe hacerse la educación: ¿Qué es una emergencia tecnológica? y ¿cómo puede estudiarse? Estas dos preguntas se colocan, de inicio, en el orden de lo empírico, en tanto experiencia social; sin embargo, lo más relevante es preguntarse: ¿Qué es una emergencia?. La cuestionante se sitúa en el orden de lo epistémico y, con mayor precisión, de lo abstracto. Es desde esta perspectiva donde se puede responder no sólo la primera pregunta -la emergencia tecnológica-, sino a todos los escenarios de significación donde se vuelque una emergencia social.

\section{Hacia una epistemología de la emergencia: una perspectiva transversal}

Una emergencia social conlleva a un quiebre o ruptura en los estatutos de la vida cotidiana, su propio dinamismo apunta a un movimiento social, entendido éste no como un proceso revolucionario, sino como la alusión a un cambio urgente del escenario social. Su inmediatez lo hace elusivo, transgresor y/o violento. Acorde con las circunstancias de lo emergente, los quiebres sociales implican un tratamiento, pero también su compresión. Es decir, los estudios de la emergencia ponen en jaque a los conceptos y metodologías tradicionales y colocan a las comunidades de estudio en perspectivas renovadoras del conocimiento.

El estudio de una emergencia social es el estudio del significado en los diferentes contextos, entendiendo que estos se producen en una comunidad social usuaria del lenguaje. De ahí la importancia de entender el marco de las significaciones que se producen en un acontecimiento. Sólo entendiendo las reglas, el uso y el contexto en que se dan (Wittgenstein, 2010), se pueden comprender las circunstancias del acontecimiento.

Tenemos que aceptar el heteromorfismo de los juegos del lenguaje con sus diferencias, inestabilidades, antagonismos, discontinuidades y paradojas. No se pueden establecer unidades por encima de la pluralidad de los juegos del lenguaje que se derivan de la pluralidad de las formas de vida. No puede existir un metadiscurso omnicomprensivo, una metateoría o fundamentación última, ya que estamos rodeados de las determinaciones estructurales, libidinales y lingüísticas (Martínez 2012: 221).

La discusión nos lleva a una noción de emergencia que trasciende a lo reciente o a lo que simplemente surge, para connotarla como una irrupción, un imprevisto detonante de una condición crítica que debe ser atendida, y reclama saberes que le brinden respuestas regeneradoras para transformar el contexto donde emergen. Sin embargo, su abordaje va más allá de los cánones tradicionales. 
Una emergencia exige atender sin demora a la población, con soluciones socialmente viables. Patrick Charaudeau, menciona que "todo análisis de un fenómeno social necesita referirse a un marco conceptual conformado por una serie de principios fundadores, hipótesis generales, conceptos que permiten construir el fenómeno como objeto de estudio, esta discusión sólo es posible si se sitúa dentro del marco que las produjo" (2009: 2).

El ámbito de las interdisciplinas apunta a desglosar los problemas emergentes en miradas transversales; lo cognitivo determina el mismo grado de corresponsabilidad con lo ético, estético y político. Ello estrecharía dos ángulos de la vida social que estarían impregnadas por la comprensión y sensibilidad de nuestro tiempo (Martínez 2012); es decir, el pensar y sentir marcarían los ejes coherentes de respuestas a la emergencia.

De fondo, significa deconstruir el discurso jerárquico del conocimiento, y sus valores, y abrir un horizonte hacia la comprensión del cambio, "de lo fragmentario, de lo local, de su énfasis en la subjetividad y la experiencia estética (Martínez 2012: 212). Esto enfatiza con mayor claridad la comprensión del estudio de la emergencia, que apuntaría no propiamente a un método, sino a la episteme que lo define.

Lo anterior significa adentrarse en un horizonte aún borroso o incierto, donde los marcos teóricos y metodológicos se tornan en trazos esperanzadores. "Se trata de aproximarse a un modo propio y peculiar, que tiene un grupo humano, de asignar significado a las cosas y a los eventos, es decir, en su capacidad y forma de simbolizar la realidad" (Martínez, 2012: 228).

Esto mismo otorga otra fisonomía a los estudios de la emergencia, cuya compresión de su episteme como objeto de estudio convoca a la diversidad disciplinaria. Charaudeau, explica que los fenómenos dados en las ciencias humanas se advierten con perspectivas interdisiciplinarias. Él mismo propone un modelo genérico sobre esas perspectivas (Ver ilustración 1).

\section{llustración 1. \\ Modelos de análisis de Patrick Charaudeau (2009)}

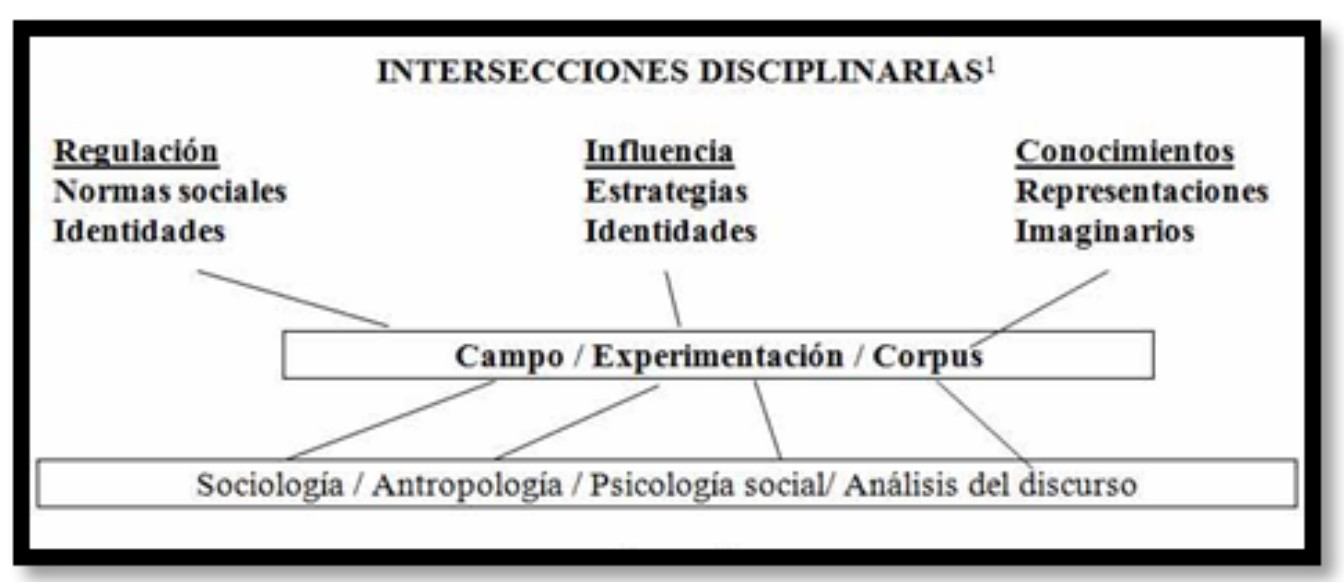

Sería perjudicial para la comprensión de los fenómenos estudiados que los defensores de una disciplina sostuvieran una actitud negacionista en relación con las disciplinas conexas 
que generan análisis sobre los mismos fenómenos: ningúno pertenece de manera exclusiva a una disciplina, y ninguna puede pretender agotar por sí sola la comprensión del fenómeno. (2009: 3). Ello conduciría las reflexiones de la emergencia no solo en el ámbito de las interdisciplinas, sino también de las transdisciplinas. Acercarse entonces a la comprensión de una emergencia demarca algunos aspectos claves que pueden ir definiendo sus trazos epistémicos.

\section{a) Ciudadanía y conflicto emergente}

En esta perspectiva lo emergente infiere una condición que emerge, que se visibiliza o que muestra que las cualidades o propiedades de un sistema se puedan inhibir, perder o dislocar. (Gascón y Cepeda, 2014). Se entiende como la irrupción del conjunto de unidades sociales dentro de un territorio. Se rompe con la articulación de flujos de mercancías, símbolos, personas, acciones, que deslizan el problema hacia la localización del nudo o rompimiento. La metáfora del movimiento social se visibiliza en el correr de todos, de un lado a otro, sin ruta fija y tropezando o chocando. Se dibuja una crisis que estropea el marco social de incidencia. La crisis se explica como un estado de cosas que tiende a desaparecer, pero también empieza a surgir otro estado de cosas. En los momentos de claroscuros entre uno y otro aparece la anomalía. El conflicto deriva entonces como un grado de desorden -frente al orden- en la incertidumbre, lo que se concreta también como un rompimiento o transgresión a la norma.

\section{b) Significación inicial del acontecimiento}

En esta arista se permite un acercamiento funcional a la crisis, detonante de un conflicto. Sin embargo, los aspectos fundamentales deben empezar a tomar la forma de una complejidad que aglutina una arquitectura lingüística compleja; su incursión a la comprensión de estos lenguajes requiere de una transversalidad de la emergencia, misma de la que se hacen cómplices las distintas disciplinas, pero su perspectiva se enfoca a través del lenguaje. Lo que ocurre es una transformación de un orden de cosas o circunstancias que se define como un acontecimiento y que Jodelet (2008) describirá como una irrupción inesperada en un orden temporal y un estado de cosas.

Los fenómenos sociales pueden considerarse, desde el punto de vista de su manifestación, como conjuntos de signos cuya organización procede de actores sociales que actúan en interacción con otros actores. Al hacerlo, estos signos se inscriben, a la vez, en procesos (los sujetos buscan influirse e individualizarse) y en estructuras, dado que estos procesos tienden a estabilizarse, y los signos a organizarse en sistemas (Charaudeau, 2009: 3). La alteración en este conjunto de signos estables y constantes avizora el territorio de lo emergente; sus quiebre pueden verse en:

- Cambio en los soportes físicos. Establecer un diagnóstico de las consecuencias materiales e impacto que la emergencia tiene en el espacio social.

- Transformación de la esfera social y cotidiana. Una de las formas visibles 
que la crisis detona en la vida social, son los cambios en la rutina de las comunidades.

- Alteración y recurrencia de los vínculos institucionales. El impacto de la emergencia puede comprenderse en los disruptivos que provoca en la interacción de los ambientes sociales. Su manifestación más elocuente se da en el rompimiento de la regla social, donde se mira con nitidez el nudo o el conflicto social. Se recurre al marco delimitativo de las instituciones sociales.

\section{c) Revisión epistémica}

Charaudeau apunta la necesidad de describir y comprobar para la deconstrucción del objeto de análisis, y su reconstrucción en categorías que supuestamente den cuenta de un determinado funcionamiento del fenómeno estudiado (2009: 2)

Emergen entonces las dimensiones de análisis con marcos de estudio que interactúan, y que hacen necesaria la incursión interdisciplinara. El enfoque de estas dimensiones alude a corpus de informaciones que constituyen parcialmente campos de acción. La manera de intervenir en ellas comunicativamente es a través de la mirada significativa que dota el estudio del lenguaje y sus discursos, no sólo en sus diversas textualidades, sino también de los sentidos que se ponen en juego; es decir, el análisis entra en el territorio de los signos y los símbolos. Su desconstrucción se hace ineludible. (Véase Tabla 1).

- Dimensión política. Este es un campo donde la norma o reglas sociales constituyen la regulación de las interacciones de los actores sociales. Los rompimientos de la regla detonan el conflicto social.

- Dimensión ideológica o de poder. Alude directamente a los actores sociales, producto de sus identidades y roles sociales. Es desde los actores donde se mide la correlación de fuerzas. La resistencia es convocada como un acto de poder.

- Dimensión epistémica. El lenguaje que transita en los colectivos o comunidades da cuenta de imaginarios que se hacen performativos en las prácticas culturales. En estas representaciones se alude a los saberes propios.

- Dimensión estética. Es un campo de interacción que se visibiliza en intersubjetividad de sujetos a través de sus sensibilidades y emociones. La puesta de los sentidos significa una condición latente del sujeto en un colectivo.

- Dimensión histórica. Se ponen de manifiesto a través de la memoria social; se constituye en la identidad colectiva de las comunidades, aluden a la tradición sobre las que se cohesiona y se blinda un colectivo social. 


\section{Tabla 1}

\begin{tabular}{|c|c|c|}
\hline \multicolumn{3}{|c|}{ DIMENSIONES ANALÍTICAS PARA EL ESTUDIO DE LA EMERGENCIA } \\
\hline DIMENSIONES & $\begin{array}{l}\text { CAMPOS DE } \\
\text { INTERACCIÓN }\end{array}$ & SISTEMAS DE LA SIGNIFICACIÓN \\
\hline Política & Normativo & $\begin{array}{l}\text { Reglas o normas que facultan y } \\
\text { regulan el marco social en el que se } \\
\text { insertan. }\end{array}$ \\
\hline $\begin{array}{l}\text { Ideológica o de } \\
\text { poder }\end{array}$ & Social & $\begin{array}{l}\text { Roles e identidades sociales en las } \\
\text { correlaciones de fuerzas. }\end{array}$ \\
\hline Epistémico & Cognitivo & İmaginarios y prácticas culturales. \\
\hline Estético & Sensitivo & $\begin{array}{l}\text { Emociones que transitan en las } \\
\text { intersubjetividades. }\end{array}$ \\
\hline Histórica & Memoria social & $\begin{array}{l}\text { Imágenes, documentos o archivos } \\
\text { que brindan la cohesión social. }\end{array}$ \\
\hline
\end{tabular}

Elaboración propia.

Estas dimensiones aluden a marcos de significación que posibilitan la necesaria incursión de otras disciplinas que convergen en el lenguaje social. Las ausencias de un método propio para todos los escenarios apuntan a la elaboración de una heurisis metodológica.

\section{d) Interpretación del hecho o acontecimiento}

Charaudeau asienta rigorosamente que en este proceso "es necesario poder interpretar los resultados de los análisis. Ahora bien, la interpretación es una operación delicada que sólo puede hacerse a posteriori, incluso cuando en un procedimiento más experimental se han postulado hipótesis previas. Interpretar consiste en una operación que relaciona los resultados de un análisis con presupuestos teóricos" (2009: 3).

Sin embargo, los procesos de interpretación no son sólo estas articulaciones de resultados con los presupuestos teóricos, que también implican seguir y significar los signos permanentes o duraderos del acontecimiento con el surgimiento de nuevas significaciones. El recorrido aparece entonces como una "arqueología de los saberes" que produce el acontecimiento.

\footnotetext{
...no es ni sustancia, ni accidente, ni calidad, ni proceso; el acontecimiento no pertenece al orden de los cuerpos. Y sin embargo no es inmaterial; es en el nivel de la materialidad, como cobra siempre efecto, tiene su sitio, y consiste en la relación, la coexistencia, la dispersión, la intersección, la acumulación, la selección de elementos materiales; no es el acto ni la propiedad de un cuerpo; se produce como efecto de y en una dispersión material. (Foucault 2007).
} 


\section{e) Énfasis creativo}

Cuando se habla sobre este punto quizá sea recomendable detenerse en las palabras de Jacques Derrida, al retomar a Levi-Strauss, y admitir que todo discurso finito está sujeto a un cierto <<bricolage>>; sobre esa instancia Derrida abre una zona más profunda del análisis del signo y plantea la noción de bricoleur:

Éste es aquel que utiliza "los medios de a bordo», es decir, los instrumentos que encuentra a su disposición, que están ya ahí, que no habían sido concebidos especialmente con vistas a la operación para la que se hace que sirvan, y a la que se los intenta adaptar por medio de tanteos, no dudando en cambiarlos cada vez que parezca necesario hacerlo, o en ensayar con varios a la vez, incluso si su origen y su forma son heterogéneos, etc. (Derrida, 1989: 6).

Al respecto, el análisis no plantea alternativas inferibles de antemano, expone en cambio, ensayar respuestas que ofrezcan salidas posibles objetivas y sustantivas a tono con el acontecimiento.

\section{f) Organización}

Los resultados se piensan como acciones convergentes, cuyas explicaciones atañen a la comprensión de significaciones subjetivas y objetivas, pero también a la totalidad de la experiencia sobre la que se produce el acontecimiento, es decir, la instancia del interpretante de la realidad. En rigor, se trata de elaborar respuestas coyunturales a situaciones complejas. La crítica y análisis prevé, en lo posible, otras alteraciones para evitarlas en consecuencia.

\section{Metodología}

La perspectiva de la emergencia demanda la innovación de la práctica educativa, misma que coloca a la investigación-acción como un proceso relevante donde el profesor se pone en contacto con los objetos de investigación. Rodríguez Gil, y García (1999) explican esta ruta de vital importancia cuando ponderan los problemas que surgen de la práctica educativa, sobre los que se torna necesario reflexionar. Ellos citan a Kemmis (1988) "La investigaciónacción es una forma de investigación llevada a cabo por $<<$ prácticos >> sobre su propia práctica" (1999: 52).

Los autores hacen particular énfasis en la investigación-acción del profesor, y consideran las características enunciadas por Elliot (1990). Dos aspectos se retoman de éstas:

- El propósito es que el profesor profundice en la comprensión de su problema. Por lo tanto, adopta una postura exploratoria frente a cualquier definición inicial de su propia situación.

- La investigación-acción adopta una postura teórica según la cual la acción emprendida para cambiar la situación se suspende temporalmente hasta conseguir una comprensión más profunda del problema práctico en cuestión (Rodríguez et al., 1999: 52). 
En este proceso de interacción con el curriculum, se identifican algunos vacíos que tienen las formaciones en comunicación, así como la definición de nuevos perfiles que demandan los planes curriculares. Este mundo complejo y emergente detecta la necesidad de contextualizar los nuevos escenarios. La perspectiva de lo emergente es una opción, es una revisión documental que se propone develar hacia dónde va la tendencia de la emergencia.

\section{Conclusión}

Sobre los tonos apocalípticos, la emergencia no puede verse sólo como "remedio" que palie las urgencias sociales. Se trata también de llegar a una conciencia social de lo que se tiene. Dice Marc Augé que muchas civilizaciones construyeron su cultura cuando miraron el paisaje de sus propios restos (2003); construir una mirada hacia los paisajes que dan cuenta de las ruinas; es decir, se trata de tomar conciencia de las urgencias para reedificar el porvenir.

Los aportes de los estudios ambientales se convierten en una contribución sustancial para entender la emergencia social respecto del medio ambiente; un entorno complejo que ha llevado a quienes estudian el tema a generar diversas arquitecturas de estudio, con las cuales se intenta brindar respuestas posibles a la emergencia social.

Estos estudios han develado como conceptos obligatorios para el mundo y su entorno ambiental a: la ética, la otredad, la diversidad, la diferencia, la comunalidad, la justicia y la solidaridad, entre otros. Desde estos ámbitos se ha entendido que los problemas emergentes del mundo están obligados a estudiarse con las muchas miradas del conocimiento, pero siempre trazados en la conciencia de lo humano.

"La construcción del mito del sujeto individual como principio de habla, del pensamiento, del sentimiento y de la acción nos han hecho olvidar su origen social, y en última instancia la fuente comunal de donde brota la conciencia individual” (Leff, 2002: 394)

La epistemología para la emergencia, comienza por quitar las aporías científicas arraigadas y abre brecha en un territorio desconocido o incierto, donde las esperanzas se focalizan en el horizonte de todos los saberes. Los alcances comprensivos de una epistemología para la emergencia serán dados en la objetivación y sensibilidad profunda que se tenga de las experiencias de vida. Como bien menciona Enrique Leff, no se trata sólo de conocer, sino de crear formas de comprensión y sentidos existenciales (2002).

La emergencia es un concepto que si bien explora alternativas a las nuevas condiciones de la realidad social, también es cierto que de manera natural no adivina los derroteros de la sociedad, aunque busque anticipar los riegos, planteando las vulnerabilidades.

El rumbo inmediato se orienta a crear una cultura para atender la emergencia; pero también está develando el proceder humano prioritariamente en la instancia de la ética, un rumbo que está poniendo en el debate los mecanismos formativos de la educación y la tecnología sobre razones más profundas como la sobrevivencia.

“...comprender la aparición y la génesis de la conciencia científica mediante un análisis de la esencia del conocimiento de sí mismo" (Maceiras, Trebolle, 1990: 42). 
La discusión sobre la emergencia entonces no es un aspecto que atañe solamente a la educación y a la tecnología, sino que es concebida como una perspectiva desde la cual es importante reflexionar lo que se origina en estos contextos, pero no sólo en ellos, sino en todos aquellos que ahora mismo están siendo develados como críticos. Sin embargo, la educación y la tecnología como escenarios de significación que permean en múltiples ámbitos haciéndolos convulsos, pueden ser punta de lanza para empezar a deliberar en relación a los estudios de la emergencia.

\section{Referencias}

Augé, M. (2003). El tiempo en ruinas. España: Ed. Gedisa.

Baudrillard, J. (2010). La ilusión vital. México: Siglo XXI.

Charaudeau, P. (2009) Análisis del discurso e interdisciplinariedad en las ciencias humanas y sociales. En Puig L. (ed.). El discurso y sus espejos, Universidad Nacional Autónoma de México, México. http://www.patrick-charaudeau.com/Analisis-deldiscurso-e.html Recuperado 23/07/2020.

Derrida, J. (1989). La estructura, el signo y el juego en el discurso de las ciencias humanas. Conferencia pronunciada en el College international de la Universidad Johns Hopkins

(Baltimore) sobre «Los lenguajes críticos y las ciencias del hombre», el 21 de octubre de 1966 (La escritura y la diferencia), Barcelona: Anthropos. http://mercaba.org/SANLUIS/ Filosofia/autores/Contempor\%C3\%A1 nea/Derrida/La\%20estructura.pdf. Recuperado 20/06/2020.

Foucault, M. (2007). La arqueología del saber. México: Siglo XXI

Gascón, P; Cepeda, JL. (2014). Pensar en la complejidad con Edgar Morin: los sistemas y hologramas. Coord. Gascón, P; Guerra, MR; Vizcarra, I; en Reflexiones en torno a la complejidad y la transdisciplina. México: UAM-UAEM

Giddens, A. (2007). Un mundo desbocado, los efectos de la globalización en nuestras vidas. México: Taurus.

Gros, B. (2008). Aprendizajes, conexiones y artefactos. La producción colaborativa del conocimiento. Barcelona, España: Editorial Gedisa.

Jacques, D. (2003). La revolución técnica. Ensayo sobre el deber de la humanidad. México: Jorale Editores.

Jodelet, D. (Septiembre, 2008) El movimiento de retorno al sujeto y el enfoque de las representaciones sociales, en Cultura y representaciones sociales. Año 3, núm. 5.

Leff, E. (2002). Saber ambiental, sustentabilidad, racionalidad, complejidad, poder. México: Siglo XXI.

Lizarazo, D. (2007). La sociedad eléctrica. Preguntas por la educación en el mundo. México: 
SEP. Col. Tecné y Pedagogía. Textos breves de investigación y comunicación educativa.

Maceiras, M; Trebolle, J. (1990). Hermenéutica contemporánea. Bogotá, Colombia: Cincel.

Martínez, M. (2012). El paradigma emergente. Hacia una nueva teoría de la racionalidad científica. México: Trillas.

Morin, E. (1991). El método I, ideas. España: Cátedra-Teorema

Rodríguez, G; Gil J; García, E. (1999). Metodología de la investigación cualitativa. Málaga: Aljibe.

UJAT (2010). Plan de Estudios de la Licenciatura en Comunicación. Universidad Juárez Autónoma de Tabasco. Villahermosa, Tabasco. http://www.archivos.ujat.mx/2014/DAEA/ pagina \%20nueva/planes $\% 20$ de\%20estudio/Sintesis $\% 20$ Comunicacion $\% 202010$. pdf. Recuperado el 24/08/2020.

UJAT (2018) Plan de Estudios de la Licenciatura en Comunicación. Universidad Juárez Autónoma de Tabasco. Villahermosa, Tabasco. http://www.archivos.ujat.mx/2019/divdaea/ Gu\%C3\%ADas\%20B\%C3\%A1sicas\%20-\%20Nuevo\%20Ingreso/GuiaBasica \% 20 Comunicacion.pdf. Recuperado 24/08/2020.

Wittgenstein, L. (2010). Investigaciones filosóficas. México: Instituto de investigaciones filosóficas. Universidad Nacional Autónoma de México, Crítica.https://filosofiaum.files. wordpress.com/2017/05/wittgenstein-investigaciones-filosoficas1.pdf Recuperado 20/06/2020.

\section{Notas}

[1] Desde la perspectiva de la complejidad Edgar Morín explica las emergencias cuando dice que los elementos que integran un sistema sufren ganancias y pérdidas cualitativas en el proceso que los transforma en partes de un todo. Se explica así un principio sistémico: todo lo que forma transforma Los elementos que integran un sistema sufren ganancias y pérdidas cualitativas en el proceso que lo transforma en partes de un todo. Se explica así un principio sistémico: todo lo que forma transforma (Morín, 1993:139).

\section{Para citar este artículo}

Pérez, F., Castillo, R., Fabila, A. (2020). Hacía una epistemología de la emergencia tecnológica y de la educación. revista luciérnaga comunicación. vol. 12 núm. 24. Pp:40-57. https://doi.org/:10.33571/revistaluciernaga.v12n24a2

Ojs: https://revistas.elpoli.edu.co/index.php/luc/issue/archive

Link: https://www.politecnicojic.edu.co/index.php/revista-luciernaga 